\title{
Desmoplastic ameloblastoma in the mandible: A case report
}

\author{
Sandeep Singh Bhatia ${ }^{1}$, Arushi Pandey ${ }^{2, *}$ \\ ${ }^{1}$ Senior Lecturer, ${ }^{2} \mathrm{HOD},{ }^{1}$ Dept. of Orthodontics and Dentofacial Orthopaedics, ${ }^{2}$ Dept. of Oral Pathology, Microbiology and \\ Forensic Odontology, ${ }^{1}$ Daswani Dental College, Kota, Rajasthan ${ }^{2}$ College of Dental Science \& Hospital, Indore, \\ Madhya Pradesh, India
}

*Corresponding Author:

Email: roshipandey@yahoo.co.in

\begin{abstract}
Introduction: $0.9-12.1 \%$ of all cases of ameloblastoma account to be Desmoplastic Ameloblastoma (DA). The presentation of the tumor is often similar to benign fibro-osseous lesions both clinically as well as radiographically. Histologically, DA is made up of small nests and strands of odontogenic epithelium which seem to be compressed by the highly collagenized stroma. We report a case of DA in a 44-year-old female with a mildly painful swelling in the anterior region of mandible. The lesion had a multilocular appearance on both panoramic radiographs as well as in the computed tomography scan. Histologically, the lesion was confirmed to be a case of DA. Regardless of the fact that DA is a rare entity, its aggressive nature binds the clinician to be cautious enough to include DA in the differential diagnosis of any lesion/growth clinically and radiographically mimicking a benign fibro osseous lesion.
\end{abstract}

Keywords: Ameloblastoma, Desmoplasia, Mandible.

\section{Introduction}

The most common classification of ameloblastoma is on the basis of its histological presentation, as being plexiform, follicular, desmoplastic, granular cell type, basal cell type, clear cell type and acanthomatous. ${ }^{1}$ In the year 1984, Eversole documented several cases and gave a detailed description of DA. He coined the term "ameloblastoma with pronounced desmoplasia" for it. ${ }^{2}$

The paucity of literature for this lesion can be understood by the fact that Eversole described less than 70 cases and Waldron and El Mofty studied about 116 cases of DA. Through the literature, it is stated that about $9-12.1 \%$ of all the reported cases of ameloblastoma fall under the DA subtype. ${ }^{3}$

DA neither has a classical clinical appearance nor is its radiological picture very pathognomonic. Radiographically, it ranges from being an irregular, multilocular radiolucency with indistinctive margins, to a combination of radiopaque-radiolucent appearance with undefined borders resembling a fibro-osseous lesion. The lesion may also present as a large expansile osteolytic lesion with mottled, multilocular or honey comb pattern. ${ }^{4}$ Tooth displacement is invariably present. $^{5}$

The only feature typical of DA is its histological picture of, dense collagenization with compressed nests and strands of cells of odontogenic origin of varying shapes and sizes. Few cases have reported metaplastic bone formation. ${ }^{6}$

Here we report one such classical case of DA in a middle aged female arising in the anterior portion of the mandible.

Clinical Presentation: A 44-year-old lady came to our dental hospital with a swelling in the chin area. She mentioned the swelling increased gradually in size over a period of 5 years and was associated with mild pain.
Extra orally, facial asymmetry was evident. The swelling was present on the right side of the face near the chin region, oval in shape and mildly tender on palpation. The overlying skin appeared slightly stretched. No sinus opening was present and no lymph node involvement was observed.

Intra-orally a large swelling approximately $5 \times 4 \mathrm{~cm}$ in size was evident, extending from the lower right second premolar to the left central incisor. The buccal cortical plate was enlarged, whereas the cortical plate of the lingual side in the affected region seemed normal. The swelling occupied the buccal vestibular area. It had a smooth surface with normal overlying mucosa. The swelling was found to be firm, bony hard, mildly tender, non-fluctuant, non-compressible and non-pulsatile on palpation Fig. 1.

The teeth in the area of the lesion were non-tender and vital; there was slight mobility of 41 . Radiographic examination (OPG) of the mandible revealed a mixed radiolucent radio-opaque lesion with unclear margins extending from the mesial surface of 45 to the mesial side of $31(5 \times 4 \mathrm{~cm}$ in size $)$. The lesion resulted in the displacement of the roots of right mandibular canine and first premolar. Though there was no sign of root resorption, the periodontal ligament space was enlarged and the lamina dura around the involved teeth was lost Fig. 2. Computerized tomography scans revealed a multilocular lesion of the size of $5 \mathrm{~cm}$ medio-laterally, $4 \mathrm{~cm}$ supero-inferiorly and $2.5 \mathrm{~cm}$ antero-posteriorly Fig. 3.

Incisional biopsy was performed and the specimen was processed. The microscopic examination of the $\mathrm{H}$ $\&$ E stained sections exhibited irregular odontogenic epithelial islands consisting of peripheral cuboidal cells which were compressed in the extensively desmoplastic stroma. Fig. 4. The above histological picture were 
suggestive of DA. The patient was referred to higher centres for treatment and follow up.

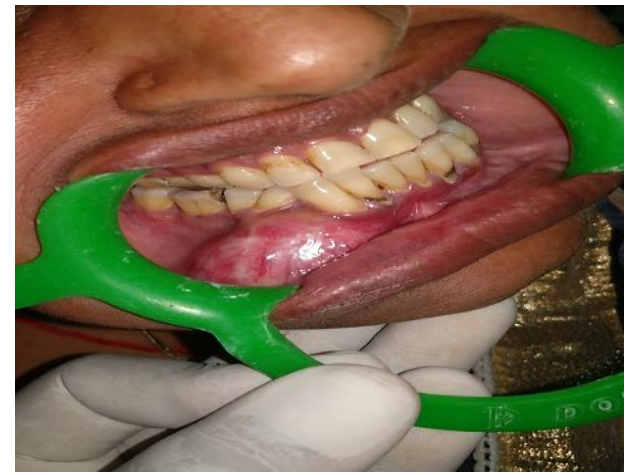

Fig. 1: The swelling had a smooth surface with normal overlying skin but stretched. On palpation, the swelling was found to be firm, bony hard in consistency, mildly tender, non-fluctuant, irreducible, non-compressible and non-pulsatile

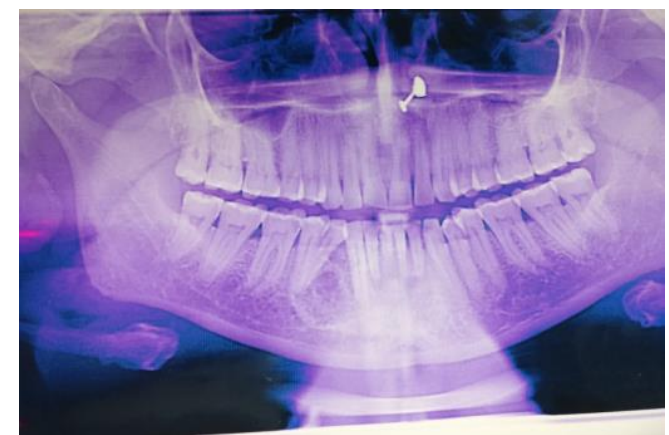

Fig. 2: The orthopantomograph revealed a diffuse ill-defined mixed radiolucent radio-opaque lesion extending from mesial surface of 45 to the mesial surface of the 32 with an approximate size of $5 \times 4 \mathrm{~cm}$. The lesion resulted in the displacement of the roots of 44 and 43 without any signs of root resorption. There was loss of periodontal ligament space on the involved teeth. There was loss of lamina dura around the involved teeth

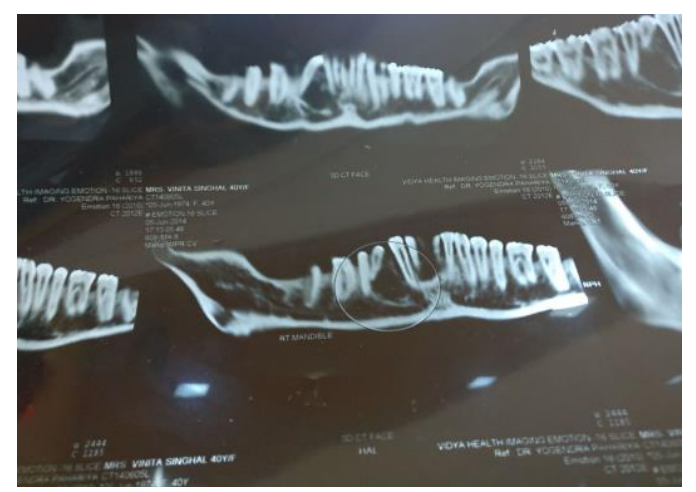

Fig. 3: Computerized tomography (CT) showed a multiloculated lesion; $5 \mathrm{~cm}$ mediolaterally, $4 \mathrm{~cm}$ superoinferiorly and $2.5 \mathrm{~cm}$ anteroposteriorly. Areas of calcification were present within the lesion giving it a soap bubble appearance.

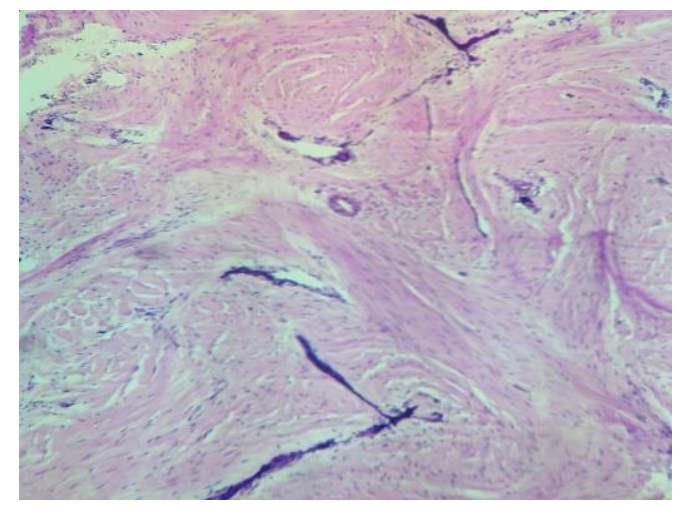

Fig. 4: Photomicrograph (Haematoxylin and eosin stain, 10x): Extensive desmoplasia was seen throughout the stroma compressing the epithelial islands

\section{Discussion}

Demographic presentation: Tumor presents a strong propensity for the anterior region of the either jaws. ${ }^{7}$ The present case also had the lesion in the anterior mandible and premolar region, which is consistent with that reported in the literature. ${ }^{8}$ The age of our patient (44 years) was in the predilection age of DA which is in the 4 th or 5 th decades of life. It has no sex proclivity. ${ }^{9}$

A painless swelling of the jaw bone usually brings the patient to a clinician. The tumor size generally ranges between 1.0 and $8.5 \mathrm{~cm}$. Tooth displacement is found in $92 \%$ of the cases, whereas only $33 \%$ of the cases show root resorption. ${ }^{10}$ In this report also, the patient described a mildly tender swelling with radiographs revealing displacement of the adjacent teeth 43 and 44 . However, no root resorption was evident.

Origin of the lesion: Kishino et al. ${ }^{11}$ identified oxytalan fibers in the connective stroma of DA with the help of potassium monopersulfate-aldehyde fuchsin stain. He proposed that these fibres which were present in the periodontal membrane could be the source of origin of DA. While others suggested the rests of Malassez present in the periodontal membrane to be the source of origin of DA. ${ }^{12}$

Radiographic features: Radiographically, nearly $50 \%$ of the DA cases have a mottled, mixed radiolucency/radiopacity with unclear margins, thus it is difficult to differentiate it from a fibro-osseous lesion. The infiltrative nature of DA involving the bony trabeculae has been hypothesized to be the reason for this radiographic picture. The literature suggests three radiological presentations of DA which can be categorized as:

1. Type I- the most common variety (osteofibrosis type) which has a mixed radiolucent-radiopaque appearance.

2. Type II- has a completely radiolucent picture (radiolucent type).

3. Type III- the rarest kind has mottled look combined with a huge radiolucent change (compound type). 
Radiographic picture of our case was consistent with that of Type 1 .

Lack of typical radiographic findings in our case, caused some difficulty in identifying it correctly. We also suspected a fibro-osseous lesion based on the radiographic findings.

Histological picture: The final diagnosis of DA is always based on histopathological findings, which is the only pathognomonic feature for DA. The usual microscopic features can be enumerated as:

An extensive stromal desmoplasia with abundance of collagen and moderate amount of cellular connective tissue, is the most consistent and distinguishing feature.

Sharp, star shaped (stellate) or "kite-like" look of the tumor islands.

Peripheral layer of the islands is usually cuboidal and occasionally hyperchromatic.

The islands may contain spindle-shaped or squamoid epithelial cells in the center. Metaplastic osteoid trabecular (osteoplasia) formation may be present.

The palisading pattern of odontogenic epithelial cells as seen in conventional ameloblastoma is absent. Myxoid changes of the juxtaepithelial stroma are often present. Our case, showed all the above mentioned features. Savithri et al. suggested that if tumor cell stimulation of connective tissue fibroblasts caused desmoplasia, they could also influence another cell type, i.e., osteoblasts to get stimulated causing osteoplasia.

Aggressiveness: DA in the maxilla vs DA in the mandible: Philipsen et al. suggested, desmoplastic ameloblastomas originating in the maxilla are more aggressive than those originating in the mandible. The closeness to vital structures as well as the very thin cortical bone of the maxilla being a weak barrier favours the spread of tumors. ${ }^{2}$

Histological dilemmas: The tumor might be misdiagnosed as another odontogenic tumor histologically as areas with only narrow strands of epithelial cells within desmoplastic stroma may look similar to odontogenic fibroma. Another differential diagnosis is squamous odontogenic tumor (SOT), as it may too present with a fibrotic stroma. The squamous metaplasia observed in some areas of the desmoplastic variant of ameloblastoma may simulate SOT if the palisading layer of the tall columnar cells is not seen. Other differential diagnosis are squamous cell carcinoma and ameloblastic fibroma. The distinguishing features of the former is prominent cytological atypia while the latter has a cellular stroma. $^{12}$

\section{Conclusion}

Due to scarcity of adequate cases, the true biologic profile of DA is still not well comprehended. The clinician should be alert enough to include DA in the differential diagnosis of any lesion/growth with a mixed radiolucent-radiopaque picture having unclear borders and occurring in the anterior region of the jaws. A radical approach to its treatment with regular follow up is mandatory in a case of DA.

\section{Conflict of Interest: None.}

\section{References}

1. Reichert PA, Philipsen HP. Odontogenic Tumors and Allied Lesions. London: Quintessence Publishing Co., Ltd; 2004. Benign Neoplasms and tumor-like lesions arising from the odontogenic apparatus showing odontogenic epithelium with mature fibrous stroma, without ectomesenchyme; p. 41.

2. Eversole LR, Leider AS, Hansen LS. Ameloblastomas with pronounced desmoplasia. J Oral Maxillofac Surg 1984;42:735-40.

3. Philipsen HP, Reichart PA, Takata T. Desmoplastic ameloblastoma (including "hybrid" lesion of ameloblastoma). Biological profile based on 100 cases from the literature and own files. Oral Oncol 2001;37:455-60

4. Laxmana AR, Gogineni SB, Thomas PS, Shetty SR. Desmoplastic ameloblastoma-a report of two clinical cases. Braz J Oral Sci 2010;9:137-41.

5. Effiom OA, Odukoya O. Desmoplastic ameloblastoma: Analysis of 17 Nigerian cases. Oral Surg Oral Med Oral Pathol Oral Radiol Endod 2011;111:e27-31.

6. Waldron CA, el-Mofty SK. A histopathologic study of 116 ameloblastomas with special reference to the desmoplastic variant. Oral Surg Oral Med Oral Pathol 1987;63:441-51.

7. Sivapathasundharam B, Einstein A, Syed RI. Desmoplastic ameloblastoma in Indians: Report of five cases and review of literature. Indian J Dent Res 2007;18:218-21.

8. Yazdi I, Seyedmajidi M, Foroughi R. Desmoplastic ameloblastoma (a hybrid variant): Report of a case and review of the literature. Arch Iran Med 2009;12:304-8.

9. Curran AE, Byerly PD. Peripheral desmoplastic ameloblastoma: Report of a rare case. J Oral Maxillofac Surg 2008;66:820-5.

10. Takata T, Miyauchi M, Ogawa I, Kudo Y, Takekoshi T, Zhao M, et al. Immunoexpression of transforming growth factor beta in desmoplastic ameloblastoma. Virchows Arch 2000;436:319-23.

11. Kishino M, Murakami S, Fukuda Y, Ishida T. Pathology of the desmoplastic ameloblastoma. J Oral Pathol Med 2001;30:35-40.

12. Itoh $Y$, Nakahara H, Itoh R, Ito A, Satou T. Osteoplastic ameloblastoma: A case report and literature review. Oral Surg Oral Med Oral Pathol Oral Radiol 2012;113:e23-8.

How to cite the article: Bhatia S.S, Pandey A. Desmoplastic ameloblastoma in the mandible: A case report. J Dent Specialities 2018;6(2):169-171. 